\title{
Narrativa
}

narrativa

Nuova serie

$41 \mid 2019$

Narrativa italiana degli anni Duemila: cartografie e percorsi

\section{(Non) è un paese per racconti}

\section{Giacomo Raccis}

\section{(2) OpenEdition \\ 1 Journals}

\section{Edizione digitale}

URL: https://journals.openedition.org/narrativa/354

DOI: $10.4000 /$ narrativa.354

ISSN: 2804-1224

\section{Editore}

Presses universitaires de Paris Nanterre

\section{Edizione cartacea}

Data di pubblicazione: 1 décembre 2019

Paginazione: 65-78

ISBN: 978-2-84016-350-3

ISSN: $1166-3243$

\section{Notizia bibliografica digitale}

Giacomo Raccis, «(Non) è un paese per racconti», Narrativa [Online], 41 | 2019, online dal 01 novembre 2021, consultato il 08 décembre 2021. URL: http://journals.openedition.org/narrativa/354 ; DOI:

https://doi.org/10.4000/narrativa.354

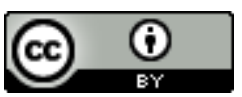

Narrativa est mise à disposition selon les termes de la Licence Creative Commons Attribution 4.0 International. 


\title{
(Non) è un paese per racconti
}

\begin{abstract}
RiASSUNTO
Contro l'adagio secondo cui i racconti non hanno mercato e in linea con la tradizione novecentesca, negli anni Zero la forma breve ha trovato nella prosa italiana una nuova centralità. In questo intervento si analizzano le forme che ha assunto nel nuovo secolo il racconto breve, in particolar modo quando organizzato nella forma-libro, a partire da testi esemplari: Personaggi precari (2007) di Vanni Santoni, per il micro-racconto in chiave umoristica; Fiction (2001) di Giulio Mozzi, per il racconto a forte statuto metaletterario; L'ubicazione del bene (2009) di Giorgio Falco, per la ricomposizione di un universo geograficamente e antropologicamente centripeto; Città distrutte (2011) di Davide Orecchio, che sul modulo biografico costruisce una raccolta a forte tenuta stilistica.
\end{abstract}

RÉSUMÉ

Contre l'adage selon lequel les nouvelles n'ont pas de marché et conformément à la tradition du $\mathrm{Xx}^{\mathrm{e}}$ siècle, dans les années 2000 la forme brève a trouvé dans la prose italienne une nouvelle centralité. Dans cet article, nous analysons les formes que prend la nouvelle dans le nouveau siècle, surtout lorsqu'elle est organisée dans la forme du livre, à partir de textes exemplaires: Personaggi precari (2007) de Vanni Santoni, pour la micro-histoire au ton humoristique ; Fiction (2001) de Giulio Mozzi pour le conte à forte composante méta-littéraire ; L'ubicazione del bene (2009) de Giorgio Falco pour la recomposition d'un univers géographiquement et anthropologiquement centripète; Città distrutte (2011) de Davide Orecchio qui sous une forme biographique construit un recueil de grande tenue stylistique.

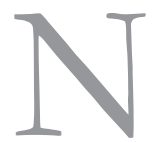

on è un paese per racconti.

Potrebbe essere questo il titolo di un immaginario film dedicato all'Italia del libro in questo primo scorcio di secolo. A fronte di una tradizione nobilissima di narrazioni brevi - dalla novella propriamente intesa fino alla short story contemporanea - che, nel solo Novecento, ha portato autori canonizzati a fare del racconto un elemento portante della propria opera, gli anni 
Duemila sembrano invece confermare una tendenza che, fin dagli anni Novanta, vede il racconto relegato in una posizione marginale del mercato editoriale, al punto da arrivare a condizionare lo stesso "orizzonte d'attesa" degli autori.

Potrebbe, si è detto. D'altra parte, è noto a tutti l'adagio secondo cui i racconti non hanno mercato ${ }^{1}$. E tuttavia, a furia di ripeterlo, questo adagio ha finito per diventare un vero topos del discorso critico, punto di partenza delle argomentazioni di quanti - per una più o meno sincera passione anticonformista - intendono ribadire l'importanza della narrazione breve nel sistema dei generi della prosa contemporanea, ma anche la sua funzione strategica in termini di mercato. Già, perché se è (quasi) vero che non esistono scrittori italiani di soli racconti, è vero anche che il racconto rappresenta ancora un genere decisivo per la collocazione di uno scrittore nel campo letterario, o quantomeno per il suo ingresso. $\mathrm{Al}$ racconto si rivolgono quei "curatori" che, attraverso lo strumento dell'antologia, tentano di fornire una campionatura attendibile di quanto di nuovo il panorama della prosa italiana propone ${ }^{2}$; con i racconti si cimentano gli scriventi in cerca di affermazione, pubblicando sulle tante riviste indipendenti - cartacee o sul web - che forniscono agli editor un bacino di pesca. E non si tratta solo di una priorità "di comodo", del racconto rispetto al romanzo, che spinge un aspirante a scommettere su una scrittura breve, in modo da ammortizzare i rischi del probabile fallimento; si tratta anche di una maggiore audacia formale e strutturale del genere breve, che consente di sperimentare portando alle estreme conseguenze un'idea o un'immagine. È anche per questo che, riprendendo le celebri posizioni di Guglielmi ${ }^{3}$, da più parti s'invoca oggi una "centralità" della forma breve - una generica forma breve - in virtù della sua "capacità di rinegoziare i confini del genere e della narrativa nella sua globalità", ma anche di condizionare gli stessi procedimenti di messa in forma romanzesca.

1. Cfr. Policastro Gilda, "Per «carenza di disorientamento». Inchiesta sulla marginalità del racconto nell'editoria contemporanea", in Allegoria, n. 69-70, gennaio-dicembre 2014, pp. 75-84: p. 75.

2. Dal progetto Under 25 ideato da Vittorio Tondelli negli anni Ottanta, l'antologia si è consolidata nel sistema editoriale italiano quale strumento di sconting efficace, confermato dalle voci riunite in raccolte come Italiana. Antologia dei nuovi narratori (a cura di Franchini e Parazzoli, 1991), Gioventù cannibale (a cura di Brolli, 1996) e fino al progetto Best off di minimum fax, editore che negli anni Duemila si è fatto carico con particolare solerzia di questo compito di ricerca.

3. Cfr. Guglielmi Guido, "Le forme del racconto", in ID., La prosa italiana del Novecento II. Tra romanzo e racconto, Torino, Einaudi, 1998, pp. 3-21: 3.

4. Tortora Massimiliano, "Il racconto italiano del secondo Novecento", in Allegoria, n. 69-70, gennaio-dicembre 2014, pp. 9-40: 40. 
Non è sempre stato così. Come ha mostrato Tortora, nel dopoguerra il racconto d'autore (Fenoglio, Moravia, Calvino, Bassani) aveva "una fisionomia molto tradizionale, recalcitrante di fronte ad ogni ipotesi di sperimentazione narrativa"; sono stati gli anni Sessanta a mutare questo equilibrio: la nuova avanguardia estende i propri condizionamenti anche alla forma breve - da Malerba a Manganelli al Calvino combinatorio - e il racconto s'impone come "laboratorio ove collaudare nuove soluzioni narrative". La brevità infatti consente di costruire testi completamente anti-narrativi o, comunque, tesi a disattendere le aspettative del lettore. E una tale attitudine si conferma - o forse addirittura si accentua - nel contesto letterario del nuovo secolo, caratterizzato dall'"imperativo del romanzo" in quanto forma espressiva di una società che vuole darsi una rappresentazione pacificata ${ }^{8}$, ma soprattutto in quanto luogo di condensazione delle molteplici manifestazioni di una narratività diffusa ${ }^{9}$. Che tuttavia trova spesso le proprie unità compositive nelle tante forme e combinazioni della narrazione breve ${ }^{10}$.

A interessare, qui, è allora il momento in cui queste combinazioni assumono forma coerente di libro. Perché, se è vero che tradizionalmente, nel dibattito teorico, si tende a contrapporre il romanzo al racconto - secondo una prospettiva di confronto tra "generi" -, non si può negare che, in un ragionamento che consideri, insieme ai caratteri strutturali, anche le dinamiche di mercato e di ricezione, la contrapposizione su cui concentrarsi dovrà essere quella tra romanzo e libro di racconti. È quindi sulla raccolta, sui criteri di selezione, sulla "combinatoria di elementi tematici e/o formali"11 e sugli altri elementi caratteristici di quello che Corti chiamava il macrotesto e che, più recentemente (e forte anche degli apporti della Short Story Cycle Theory anglosassone), Santi ha

\section{Ibid., p. 15.}

6. Ibid., p. 37.

7. Cortellessa Andrea, "Introduzione", in La terra della prosa. Narratori italiani degli anni Zero (1999-2014), a cura di Andrea Cortellessa, Roma, L’Orma, 2014, pp. 13-65: 36.

8. Cfr. Berardinelui Alfonso, Non incoraggiate il romanzo. Sulla narrativa italiana, Venezia, Marsilio, 2011.

9. SimONETTi Gianluigi, La letteratura circostante. Narrativa e poesia nell'Italia contemporanea, Roma, il Mulino, 2018, p. 23.

10. Cfr. Tirinanzi de Medici Carlo, "Breve/Lungo. Declinazioni letterarie di due radicali cognitivi", in Tirinanzi De Medici Carlo, Pradel Stefano (a cura di), Brevitas. Percorsi estetici tra forma breve e frammento nelle letterature occidentali, Trento, Università degli Studi di Trento. Facoltà di Lettere e Filosofia, 2018, pp. 7-45: 32-38.

11. Corti Maria, “Testi o macrotesto? I racconti di Marcovaldo", in Il viaggio testuale, Torino, Einaudi, 1978, pp. 184-200: 185. 
chiamato politesto ${ }^{12}$, che si dovranno appuntare le considerazioni di questo contributo. Che ha come obiettivo quello di spostare leggermente - ma in maniera si spera significativa - l'angolazione del discorso tradizionale sul racconto, aprendo il grandangolo alla progettazione macrostrutturale, alla definizione di invarianti tematiche o stilistiche, a quegli elementi, cioè, che garantiscono al testo l'“atmosfera di un'opera poetica"13, fungendo da implicita cornice ai singoli racconti.

Il corollario non secondario di questa operazione prospettica consisterà in una sintetica cartografia delle forme assunte dal racconto italiano negli anni Duemila, a partire da quattro modelli formali - incarnati da altrettante opere-campione - che, pur non esaurendo l'intero spettro, restituiscono - a parere di chi scrive - la specificità del genere breve, mostrandone la capacità di anticipare le trasformazioni che arrivano poi a toccare anche il genere lungo e soprattutto di rendere esplicite e mimeticamente evidenti alcune istanze di senso che la struttura romanzesca più facilmente diluisce e camuffa.

\section{MiCRORACCONTI}

Si potrebbe cominciare dicendo che il racconto si presta più del romanzo ad assimilare i pattern cognitivi della nuova civiltà mediatica. Quel che negli anni Novanta era lo rapping televisivo, nei Duemila diventa la navigazione in rete: il web, con le sue nuove forme testuali (blog, email, social network) e le sue logiche espressive, caratterizzate in particolar modo da sintesi e immediatezza comunicativa, ha rappresentato il maggior propellente alla sperimentazione formale negli ultimi vent'anni. Se però il romanzo può far uso di queste nuove forme ricomponendole all'interno di un insieme narrativo più ampio, reso coeso da cuciture diegetiche inserite ex novo (è il caso di Mio salmone domestico di Emmanuela Carbé, 2013, o Tranquillo Prof., la richiamo io di Christian Raimo, 2015), la raccolta di racconti può riproporle "in purezza", confidando peraltro nella capacità del libro di restituire anche il senso di infinita riproducibilità tipica della rete.

È quanto ha fatto Vanni Santoni con Personaggi precari, libro costruito a partire dai contenuti di un blog - omonimo e oggi chiuso - in cui l'autore pubblicava brevissimi e fulminanti profili di personaggi d’invenzione. Nasce così la prima

12. SANTi Mara, "Simul stabunt... Note per una teoria politestuale della raccolta di narrativa breve", in Allegoria, n. 69-70, gennaio-dicembre 2014, pp. 85-104.

13. Auerbach Erich, La tecnica di composizione della novella (1921), Roma-Napoli, Theoria, 1984, p. 24. 
raccolta, ancora "embrionale", uscita nel 2007 per RGB, seguita da quelle del 2013 e del 2017, entrambe per Voland, entrambe riviste e ampliate. Si tratta di testi che toccano una molteplicità di soluzioni stilistiche - "dalla microstoria al dialogo (brevissimo o più articolato), dall'epigramma al ritratto narrativo"14 tutte in qualche modo riconducibili al genere del microracconto.

\section{GABRIELLA}

Possibile che sia diventata una persona sciocca e cattiva? No, no: ho solo l'anima in disordine.

\section{EUGENIO}

Poche cose sono brutte quanto cercare di salire sul carro del vincitore ed esser spinto giù a calci ${ }^{15}$.

Nella nota d'apertura alla seconda edizione si legge: "I personaggi offerti da Personaggi precari sono disposti ad accettare ruoli sia primari che marginali, a tempo determinato o indeterminato, e autorizzano il datore di lavoro a disporre delle proprie prestazioni in modo assolutamente arbitrario" ${ }^{16}$. Una tale precisazione serve a fornire un'implicita indicazione di lettura per il titolo dell'opera, chiarendo che i cinquecento individui che si avvicendano nella raccolta sono innanzitutto personaggi, pronti a entrare in qualsiasi storia di qualsiasi genere. Spetterà al narratore che deciderà di farne uso dare ulteriore sviluppo ai loro profili sintetici. In questo modo acquisiscono importanza decisiva i pochi tratti forniti dai microracconti, spesso peraltro estremamente elusivi circa conformazione caratteriale e fisica dei soggetti. Ha ragione Bruni quando scrive che in queste pagine "la precarietà è una forma, molto più che un contenuto" 17 : e questa precarietà muta il segno del proprio significato, trasformandosi in valore aggregante (lasciando ogni personaggio allo stato di abbozzo, l'autore ne può accumulare uno dietro l'altro, senza soluzione di continuità) e anche creativo (giacché ogni racconto è un invito a estrarre il personaggio dalla serie per trasformarlo in protagonista di una storia).

Con questa formula Santoni denuncia senz'altro una certa parentela con gli esperimenti combinatori di Calvino o con quelli parodici di Manganelli, ma più ancora svela un aspetto decisivo del proprio background culturale, ovvero

14. Bruni Raoul, "Lo stereoscopio dei precari”, in SANTONI Vanni, Personaggi precari, Roma, Voland, 2013, pp. 154-157: 156.

15. Santoni Vanni, Personaggi precari, cit., p. 61.

16. Ibid, p. 7.

17. BRUni Raoul, "Lo stereoscopio dei precari”, cit., p. 155. 
la passione per $i$ giochi di ruolo in stile Dungeons $\&$ Dragons ${ }^{18}$ : ogni microracconto può infatti essere letto come una sintetica scheda personaggio, messa in mano a un lettore invitato a trasformarsi in ideatore di storie, che potranno, perché no, integrarsi a quelle di altri personaggi precari, andando a comporre più articolate "storie-partite precarie". L'invito è implicito, naturalmente; ma la prima apparizione di queste schede su un blog, e quindi all'interno di una realtà mediale improntata alla condivisione e alla collaborazione degli utenti, autorizza ad attribuire questo carattere a una raccolta che - nella selezione e ricombinazione dei contenuti per l'edizione cartacea - cerca di mantenere intatti alcuni caratteri dell'opera originaria. Come l'idea che la storia sia l'esito di un processo creativo in cui l'autore cede la palla al lettore, invitato a proiettarsi, con la propria immaginazione, nei bianchi tipografici che separano un racconto dall'altro; o come l'impressione di moltiplicazione infinita dei percorsi e dei personaggi che l'accumulo di questi microracconti suggerisce (testimoniato dalle tre diverse edizioni).

D'altra parte, il passaggio dalla rete alla carta è sì frutto di una selezione delle storie secondo un criterio di rappresentatività del "progetto", ma non prevede la ricomposizione dei testi in una macrostruttura nuova. È quindi la natura in qualche modo "repertoriale" della raccolta a segnare la distanza tra Santoni e le strutture combinatorie di Calvino e ad avvicinarlo invece alle latitudini accumulative del Flaiano dei "diari" o al Pontiggia delle Vite di nomini non illustri (1993). E in questo caso la linearità della struttura testuale diventa elemento caratterizzante di un'opera che si distingue per coerenza nel panorama narrativo di questi anni (anche all'interno dello stesso campo dei microracconti, che annovera autori come Franco Arminio, che con le sue Cartoline dai morti sembra seguire un percorso compositivo analogo, pur se privo di connessioni intermediali). Anche - o soprattutto - per la sua capacità di testimoniare un'epoca e la sua epistemologia: quella del flusso, delle "esistenze frammentate e attimali", delle "intensità momentanee"19, quella per cui l'unico modo per raccontare il presente non consiste nella sintesi per via di tipizzazione, ma al contrario nell'integrale enumerazione del reale, imitando l'unica opera contemporanea capace di riprodurre il mondo in scala naturale: la rete.

18. Ai quali ha dedicato il racconto-saggio La stanza profonda (2017).

19. Mazzoni Guido, I destini generali, Roma-Bari, Laterza, 2015, p. 22. 


\section{MicROCOSMI}

In una recensione a Cartoline dai morti Rossella Milone chiamava in causa, per definirne la struttura, l'immagine del caleidoscopio: "uno strumento che, servendosi di specchi e pezzetti di vetro, crea infinite strutture simmetriche di vari colori e forme" ${ }^{\prime 20}$. Vetri che offrono all'occhio immagini ogni volta differenti, ma originate dalla stessa matrice. Si potrebbe utilizzare questa metafora per descrivere il libro d'esordio di Giorgio Falco, Pausa caffe, pubblicato da Sironi nel 2004 nella collana "Presente indicativo" curata da Giulio Mozzi. 68 "pezzi" dalla configurazione eterogenea per lunghezza, impostazione diegetica e costruzione narrativa raccontano la variegata realtà di una grande compagnia di telecomunicazioni. La macrostruttura non offre altri criteri ordinativi che il semplice accumulo dei frammenti, riproducibili, nella loro diversità, secondo una logica modulare. Si capisce allora che lo specchio che ha dato origine a questa caleidoscopica rappresentazione è quello rotto del modernismo, che azzera i nessi logici e fa di ogni pezzo un frammento a sé stante, ricollegabile agli altri solo in virtù di una condivisa funzione esemplificativa. La singolarità di un personaggio o di uno sketch può essere considerata rappresentativa in virtù di un criterio non analogico, bensì statistico, essendo ciascun racconto equivalente a ogni altro nell'irriducibile molteplicità del mondo contemporaneo. La struttura del libro serve allora a mimare $e^{21}$ la frammentarietà del lavoro nel nuovo terziario, la precarietà degli impieghi, ma anche l'isolamento sociale e affettivo degli individui. Tuttavia, se il modernismo assegnava alla parzialità della rappresentazione un'indiscutibile significatività, poiché alludeva, e negativo, a una sintesi che faceva da orizzonte di senso al racconto, nel nuovo secolo non esiste alcuna totalità rappresentabile, se non per via di montaggio di esempi particolari.

A una struttura macrotestuale chiusa ${ }^{22}$ è invece riconducibile la seconda raccolta di Falco, L'ubicazione del bene, del 2009, senz'altro il suo capolavoro. Qui si rafforza il vincolo cronotopico che lega i nove racconti, tutti ambientati nella cittadina di Cortesforza, nonluogo d'invenzione, ma verosimilmente localizzato nell'hinterland milanese. La rappresentazione è puntiforme, ogni racconto

20. MiLone Rossella, "Messaggi in limine", in L'Indice dei libri del mese, n. 1, gennaio 2011, p. 18.

21. Come ha scritto Simonetti, Falco "fa della frammentazione il contenuto e insieme la forma del racconto"; cfr. SimONETTI Gianluigi, La letteratura circostante, cit., p. 75.

22. A tal punto che l'opera "si legge come un romanzo": TiRinanzi DE Medici Carlo, Il romanzo italiano contemporaneo. Dalla fine degli anni Settanta a oggi, Roma, Carocci, 2018, p. 237. 
fornisce un'immagine-campione della degenerazione psicologica e relazionale che definisce l'identità di una piccola borghesia rampante, ma psichicamente fragile. L'ansia performativa e i fallimenti brutali, le euforie dopate e i crolli nervosi, e infine la mercificazione dei corpi e dei rapporti, oltre che dei "beni" che danno il titolo alla raccolta: sono questi caratteri a regolare le relazioni tra gli individui, così come le singole vite in una realtà che dichiara il trionfo di un "realismo capitalista" 23 che dall'universo lavorativo è arrivato a colonizzare anche la vita domestica e la dimensione psichica.

Costruendo la propria raccolta intorno a un microcosmo narrativo ben definito, Falco adotta una soluzione strutturale molto frequentata nella recente narrativa italiana. Tuttavia, diversamente da altri "libri di racconti intrecciati", L'ubicazione del bene non si affida a un repertorio fisso di personaggi che ritornano di racconto in racconto (come in Cani dellinferno di Daniele Benati, 2004), né assegna a una voce narrante fissa (come in S'êfatta ora di Antonio Pascale, 2006) il compito di colmare gli spazi vuoti tra un racconto e l'altro oppure di mettere in evidenza il vettore temporale che lega i momenti topici fatti oggetto dei singoli racconti (soluzioni queste che tradiscono tutte una certa "ambizione romanzesca"). In questo caso l'elemento di coesione è dato da una complessa istanza diegetica, costruita - come ha rivelato l'autore - a partire dallo spunto fornito da una fotografia dell'americano Lewis Baltz. Si tratta di un'immagine tratta dall'album The New Industrial Parks near Irvine, California (1974) che presenta la grande vetrata a specchio di una villa, divisa in nove riquadri che riflettono, con minimi scarti dovuti ai gradi di rifrazione, il paesaggio suburbano di fronte: una strada, un capannone, alberi e colline all'orizzonte. L'unico elemento del paesaggio che viene tagliato dall'immagine sulle vetrate è la sagoma del fotografo. A questa figura corrisponderebbe appunto l'istanza narrativa dell'Ubicazione, che di preferenza si traduce in una terza persona distaccata e algida, espressione di un punto di vista "delle cose", ma che può anche incarnarsi in una prima persona. Questa infatti non dà espressione a una psicologia complessa, né mostra adesione affettiva al vissuto che racconta, ma al contrario accentua la distanza clinica, limitandosi a osservare quel che accade come se fosse l'oggetto di un esperimento scientifico: "Non voglio parlare di un matrimonio finito, questa è la storia di un luogo, il luogo che sta intorno alla casa di via Carlo Borromeo 10/E a Cortesforza, Milano"24.

È così che Falco dà rappresentazione alla profonda scissione dei soggetti, che fa da specchio all'isolamento valoriale ed esistenziale già fotografato in Pausa

23. Fisher Mark, Realismo capitalista, Roma, Nero, 2018.

24. Falco Giorgio, L'ubicazione del bene, Torino, Einaudi, 2009, p. 16. 
caffe. Le scelte narrative microtestuali - in particolare il ricorso al montaggio ${ }^{25}$ come architrave di un racconto tutto condensato in pochi emblematici momenti - si coordinano a una macrostruttura in cui ogni tassello diviene insostituibile nella composizione di una rappresentazione che, nella sua parzialità, restituisce un'immagine definitiva del microcosmo di Cortesforza.

\section{IPERTESTI}

Come detto, a tenere a battesimo Falco, e molti altri scrittori della sua generazione (Franco Arminio, Laura Pugno, Maurizio Torchio o Vitaliano Trevisan), è stato Giulio Mozzi. Maieuta dell'editoria italiana, Mozzi è forse l'unico in Italia a potersi fregiare del titolo di "autore di racconti", giacché la sua produzione di narratore si è svolta quasi interamente nel recinto delle forme brevi. Per quanto la sua opera si sviluppi principalmente negli anni Novanta, all'inizio del nuovo secolo Mozzi dà alle stampe una raccolta che coglie in maniera puntuale l'aria del tempo: del suo e dei quindici anni a venire, confermando così l'eccezionale fiuto dell'autore.

Fiction esce nel $2001^{26}$ ed è una raccolta di sedici prose divisibili in due gruppi. Nel primo si trovano sei testi accomunati dalla postura enunciativa in prima persona e dal fatto di essere tutti, in un modo o nell'altro, gravitanti intorno al tema della morte: un uomo scrive ai direttori di varie testate minacciandoli di uccidersi se non pubblicheranno la sua lettera, un professore universitario pronuncia il discorso di commiato per il proprio maestro nella cerimonia dell'alzabara. Nel secondo invece si trovano nove testi attribuiti ad autori diversi da Mozzi. La varietà testuale è maggiore, poiché, oltre ad appelli, lettere e confessioni, compaiono racconti veri e propri, ma anche un'intervista. A far da cesura ai due gruppi un testo dalla natura insolita, Narratology, "una lunga e dolorosa interrogazione sui motivi per cui negli ultimi duemila anni nessuna pagina è stata aggiunta agli scritti ispirati da Dio"27.

Tanto i testi del primo, quanto quelli del secondo gruppo sono seguiti da note che riportano rispettivamente vicende di cronaca locale che mostrano affinità con i fatti narrati (talvolta mostrandone la verosimiglianza, talaltra smentendoli) e

25. Cfr. Luperini Romano, "Il trauma e il caso. Sulla tipologia della novella moderna”, in ID., L'autocoscienza del moderno, Napoli, Liguori, 2006, pp. 163-176: 171.

26. Mozzi Giulio, Fiction, Torino, Einaudi, 2001; una nuova edizione "sfoltita e incrementata" è stata intitolata Fiction 2.0, Milano, Laurana, 2017.

27. Fiorentini Luca, "Smettere anche solo provvisoriamente di essere scrittori", in L'Indice dei libri del mese, n. 4, aprile 2018, p. 13. 
notizie biografiche sugli autori dei testi. Accade così che la confessione di chi ha ucciso il parroco che era la sua guida spirituale trovi riflesso nella vicenda di un uomo della provincia di Brescia che, nel 1998, si consegnò alle forze di polizia dopo un omicidio analogo, trascritto in un memoriale nelle ore successive al delitto; mentre la lettera con cui il poeta Lucio De Palma denuncia la parzialità del voto della giuria di un premio a cui ha partecipato è accompagnata da alcune sue poesie e da altri documenti su di lui (una targa in sua memoria, la lettera di rifiuto di un editore).

Allora, se lo spazio del testo è quello della letteratura e - giusto il titolo della raccolta - della "finzione", i paratesti intervengono a mostrare ora la somiglianza tra storie raccontate e vicende realmente accadute, ora invece a radicare nella profondità biografica dei loro autori $\mathrm{i}$ testi gravitanti intorno a situazioni simili al vero ma mai del tutto verificate. La "tentazione ipertestuale" 28 a cui queste narrazioni soggiacciono, tuttavia, serve a confondere le acque. Infatti, se le notizie di cronaca riportate nelle prime note sono vere, le identità degli autori dei racconti della seconda parte del libro sono eteronimi di Mozzi, personaggi "creati e prodotti" insieme all'amico artista Bruno Lorini, ai quali, con questi testi ma soprattutto con una serie di performance "esterne", ha fornito negli anni un'identità artistica attendibile ${ }^{29}$. Fiction si rivela così una raccolta di testi nati per depistare il lettore, mettendo in crisi i due capisaldi di ogni patto narrativo: "lo statuto finzionale delle storie e l'identità di chi narra" 30 . Testo e paratesto dialogano per comporre un mondo d'invenzione, che mostra tuttavia continue intersezioni con la realtà abitata dal lettore.

Non è un caso che, anche per molti di questi testi, la prima sede di apparizione siano stati siti e riviste in rete, luoghi in cui la proliferazione di identità fittizie e la smaterializzazione dei dati biografici consente di rendere poroso - e a tratti impercettibile - il confine tra vero e falso. Mozzi conduce questo esperimento su due distinti terreni discorsivi. Da un lato c'è il campo dell'arte, delineato attraverso la costruzione di repertori di opere e artisti fittizi alla maniera di Borges o del Bolaño della Letteratura nazista in America, ma sempre divertendosi a inserire in questi giochi testuali la propria figura, come curatore, come autore

28. Cfr. Simonetti Gianluigi, La letteratura circostante, cit., pp. 77-83.

29. Il caso più evidente è quello di Carlo Dalcielo, di cui si riporta un'intervista accompagnata da un manifesto di poetica e che nel 2008 figurerà come co-autore insieme a Raymond Carver del volume Il pittore e il pesce, effettivamente edito da minimum fax.

30. Cortellessa Andrea, "Di una funzione Mozzi", in Le parole e le cose, 9 settembre 2017, http:/ /www.leparoleelecose.it/?p=28867 
o come semplice sbobinatore. Così facendo porta avanti, per altra strada rispetto ai precedenti libri, quella "sperimentazione sull'io autoriale" che gli consente di gettare il lettore in un vero e proprio "spaesamento ermeneutico"31. Dall'altro lato c'è il campo della cronaca, continuamente chiamato a confrontarsi, attraverso le note in calce, con le storie inventate dall'autore. Si tratta di un terreno di contesa tradizionale per la letteratura, che tuttavia nel primo quindicennio di questo secolo ha trovato in Italia una nuova centralità narrativa, diventando il referente esplicito di esperimenti condotti sia sulla forma breve (Non saremo confusi per sempre di Marco Mancassola, 2011) sia su quella lunga (tra i tanti, Elisabeth di Paolo Sortino, 2011), ma volti sempre a interrogare la dimensione etica dell'individuo e la capacità della parola letteraria di "rivelare verità nascoste e ulteriori” ${ }^{\prime 2}$.

\section{Biofictions}

Dalla cronaca alla storia il passo è breve. Di formazione storico, Davide Orecchio ha dedicato il suo libro d'esordio, Città distrutte (2011), alla narrazione di Sei biografie infedeli - così recita il sottotitolo -, vite di personaggi d'invenzione ispirati a figure storicamente esistite, anche se non tutte note al grande pubblico. I protagonisti sono Kauder, Betta Rauch, Pietro Migliorisi, Valentin Rakar, Eschilo Licursi ed Éster Terracina; i loro referenti storici sono il diplomatico tedesco Wilhelm von Humboldt, la madre dell'autore Oretta Bongarzoni, il padre dell'autore e dirigente del PCI nel dopoguerra Alfredo Orecchio, il regista Andrej Tarkovskij, il sindacalista Nicola Crapsi e le migliaia di donne desaparecidas d'Argentina. Al di là di questo rispecchiamento, Orecchio costruisce queste vite attraverso una forma narrativa che ibrida il genere storico-documentario della biografia con quello prettamente letterario della short story.

Da un lato egli fa ricorso a testimonianze dirette, citazioni, rimandi bibliografici e note al testo, per dare a queste biografie lo spessore della realtà (e dei suoi discorsi). Dall'altro i riferimenti epitestuali sono sempre, in qualche modo, falsi: perché del tutto fittizi, oppure da attribuire alle controfigure storiche dei personaggi, oppure ancora manipolati rispetto agli originali. Ogni documento così ha l'apparenza della verità storica, ma le sfugge sempre impercettibilmente, rispondendo alle logiche dell'invenzione letteraria. Sono le note al testo a svelare

31. MARCHESE Lorenzo, L’io possibile L'autofiction comeparadosso del roman zo contemporaneo, Massa, Transeuropa, 2014, p. 154.

32. Mozzi Giulio, Fiction, cit., p. 5. 
l'impasto di realtà e finzione di cui sono fatti questi racconti, fornendo al lettore il "codice" per leggerli correttamente. Valga, tra tutti, l'apertura della nota agli Episodi dalla vita di Pietro Migliorisi (1915-2001): "Assegnare a ogni riga il suo autore. Chi desidera farlo per quanto scritto sopra inizi dalle virgolette «», dove i testi sono di Alfredo Orecchio"33.

Sempre sul versante letterario, poi, vanno registrate alcune strategie retoriche "poco consone al discorso storiografico, come l'insistenza sulla sfera privata" o "la resa dei pensieri dei personaggi biografati" 34 . Si può legittimamente dire, quindi, che le storie raccontate da Orecchio s'inseriscono nella tradizione delle biofictions $^{35}$, e, al suo interno, sulla scia di quegli autori (come Kiš e Michon) che hanno declinato questo genere in senso politico, affidandogli il compito di mostrare "i rapporti che legano le vite 'sconosciute' raccontate e la Storia collettiva che attraversano" 36 allo scopo di portare a galla verità sui grandi fatti del Novecento attraverso l'integrazione tra "i materiali dello storico e gli strumenti dello scrittore, ovvero l'archivio e l'immaginazione" ${ }^{\prime 37}$.

A livello di struttura del libro, se comuni a tutti i racconti sono tanto l'insistenza su uno statuto gnoseologico ibrido, continuamente ribadito dal dialogo tra testo e paratesto, quanto l'adozione del modulo narrativo della biografia, il vero elemento di coesione e coerenza è costituito dal tessuto stilistico attraverso cui le storie vengono raccontate. Orecchio mette a reazione l'impiego di un presente storico tipico delle "vite" con una focalizzazione mobile, che si sposta dal personaggio protagonista a un imprecisato punto di osservazione esterno, capace di conferire un senso di necessità alla concatenazione di eventi e micro-eventi che compongono queste biografie. E proprio la difficoltà di individuare i momenti principali di una vita, quelli che ne racchiudono allegoricamente il senso o ne segnano in maniera definitiva il percorso, è l'oggetto di alcuni squarci metanarrativi che il narratore apre di tanto in tanto nel racconto, prendendo la

33. Orecchio Davide, Città distrutte. Sei biografie infedeli, Roma, Gaffi, 2011; Milano, il Saggiatore, 2018, p. 170.

34. Pennacchio Filippo, "Allofiction. Appunti su Latronico, Siti, Orecchio", in il verri, n. 64, giugno 2017, pp. 72-88: 78.

35. Cfr. Castellana Riccardo, "La biofiction. Teoria, storia, problemi”, in Allegoria, n. 71-72, 2015, pp. 67-97.

36. Mongelli Marco, "Le biofictions di Davide Orecchio tra Danilo Kiš e Pierre Michon", in Nuova Corrente, n. 162, luglio-dicembre 2018, pp. 87-102: 89.

37. Mongelli Marco, "Riscrivere la storia per non dimenticarla. 'Mio padre la rivoluzione' di Davide Orecchio", in La Balena Bianca, 8 marzo 2018, https://www.labalenabianca. $\mathrm{com} / 2018 / 03 / 08 /$ riscrivere-la-storia-non-dimenticarla-mio-padre-la-rivoluzione-davide-orecchio/ 
parola in prima persona. L'autore sottopone così la figura dello storico agli smottamenti che provengono da un'interrogazione letteraria, che problematizza la pretesa di raccontare come sono andate per davvero le cose. Ma soprattutto, da questi passaggi si irradia nel testo un regime figurale denso, deputato a trascendere continuamente il piano letterale della storia.

Un tessuto metaforico che appare nella sua evidenza fin dal titolo, che accosta la vita dei personaggi a un paesaggio di macerie su cui l'autore interviene a ricostruire, ricomponendo $i$ frammenti in una trama che restituisca senso e memoria a storie cancellate dalla violenza del tempo. Vanno nella stessa direzione le ricorrenti immagini arboricole, che simboleggiano il lavoro di potatura e manutenzione che sulle vite opera chi se ne prende carico come narratore: "Defalco rami che s'arrampicherebbero fino a dover ricadere e penzolare e scelgo chi salvare, chi sacrificare, quale verde sopravvivrà e quale finirà in un cartoccio marrone" 38 .

Proprio la natura inevitabilmente frammentaria di queste vite impone inoltre la scelta della forma breve. Da un lato infatti, per quanto ognuna delle sei biografie potrebbe anche essere fatta oggetto di una narrazione ampia, l'intenzione di chi scrive di condensare il senso di una vita in pochi, determinati passaggi, procedendo "a balzi, per tagli, chiaroscuri violenti e cortocircuiti fulminanti", suggerisce la forma della "novella moderna" 39 . Dall'altro, il significato principale del libro risiede proprio nella declinazione plurale del suo titolo e nell'idea di una moltiplicazione delle catastrofi a partire da un comune modulo narrativo e secondo un progetto potenzialmente estendibile. Ed effettivamente esteso da Orecchio con l'ultimo libro, Mio padre la rivoluzione (2017), in cui le storie narrate insistono sulla medesima idea di riscrittura della Storia a partire da un campo discorsivo ibrido (protagonisti sono stavolta personaggi storici celebri - da Stalin a Bob Dylan a Gianni Rodari -, ma calati in situazioni ucroniche) e soprattutto su una temperatura stilistica che fa da collante all'intero volume (oltre al tono epico, allineato con la storia "grande" della rivoluzione d'ottobre, che fa da baricentro al libro, si ritrova un gusto per le metafore naturali già visto all'opera in Città distrutte).

Orecchio ha così tracciato il perimetro di una narrativa originale e attualmente unica nel panorama italiano; egli, infatti, porta alle estreme conseguenze quella propensione alla discontinuità narrativa tipica di tante narrazioni ibride - si pensi ai libri di Helena Janeczek o di Filippo Tuena - che, pur partendo da

38. Orecchio Davide, Città distrutte, cit., p. 177.

39. Luperini Romano, "Il trauma e il caso", cit., p. 175. 
premesse epistemologiche analoghe e condividendo un uso politico della memoria, cercano di cucire i frammenti del racconto all'interno di una compagine romanzesca, tenuta assieme proprio dalle manifestazioni metanarrative dell'io autoriale. Orecchio invece fa coincidere il proprio progetto con la moltiplicazione di narrazioni brevi, nella convinzione che se mai sarà possibile ricostruire per intero la Storia, riscattandola alla complessità dei suoi significati, questo accadrà non per merito di un'unica, grande narrazione, bensì per via di accumulo di storie: ciascuna esito di strategie di selezione e composizione specifiche, ciascuna portatrice di una propria verità particolare.

Giacomo RACCIS

Università di Bergamo 